\title{
ARTICLE
}

\section{TiN Paper for Ultrafast-Charging Supercapacitors}

Check for

Check for
updates

\section{Cite as}

Nano-Micro Lett.

(2020) 12:3

Received: 13 October 2019

Accepted: 17 November 2019

Published online: 10 December 2019

(C) The Author(s) 2019

\author{
Bin Yao ${ }^{1}$, Mingyang $\mathrm{Li}^{1,2}{ }^{1,}$ Jing Zhang ${ }^{1}$, Lei Zhang ${ }^{1,3}$, Yu Song ${ }^{1}$, Wang Xiao ${ }^{1}$, \\ Andrea Cruz ${ }^{1}$, Yexiang Tong ${ }^{2}$, Yat $\mathrm{Li}^{1}{ }^{凶}$ \\ $\square$ Yat Li, yatli@ucsc.edu \\ 1 Department of Chemistry and Biochemistry, University of California, Santa Cruz, Santa Cruz, CA 95064, \\ USA \\ 2 KLGHEI of Environment and Energy Chemistry, MOE of the Key Laboratory of Bioinorganic and Synthetic \\ Chemistry, School of Chemistry and Chemical Engineering, Sun Yat-Sen University, Guangzhou 510275, \\ People's Republic of China \\ 3 State Key Laboratory of Advanced Technology for Materials Synthesis and Processing, Wuhan University \\ of Technology, Wuhan 430070, People's Republic of China
}

\section{HIGHLIGHTS}

- The superior conductivity and unique porous electrode structure in the TiN paper enable fast charging by simultaneously providing efficient ion diffusion and electron transport.

- The TiN paper-based supercapacitors exhibit charging/discharging at an ultrahigh scan rate of $100 \mathrm{~V} \mathrm{~s}^{-1}$ in a wide voltage window of $1.5 \mathrm{~V}$ in $\mathrm{Na}_{2} \mathrm{SO}_{4}$ neutral electrolyte and show zero capacitance loss after 200,000 cycles.

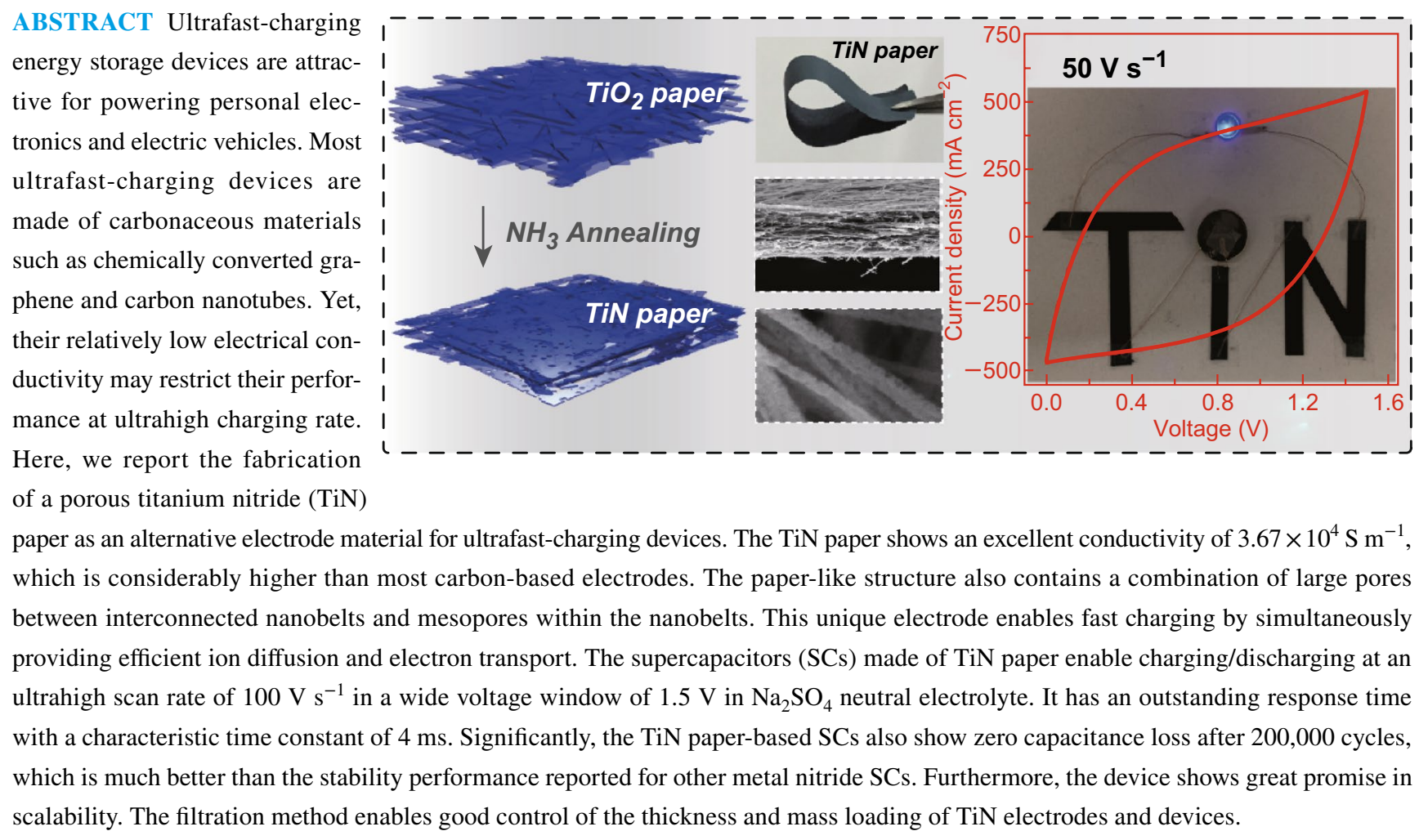

KEYWORDS Ultrafast charging; Wide voltage window; TiN; Paper-like electrode; Supercapacitors 


\section{Introduction}

The emerging advances in portable and wearable electronics urge the rapid development of fast charging and discharging energy storage devices [1-6]. Supercapacitors (SCs), renowned for their high charging rate and long lifespan, have received great attention in the past decades [7, 8]. Currently, most SCs are operated at a charging speed of $2-100 \mathrm{mV} \mathrm{s}^{-1}$, which corresponds to the charging time from tens of seconds to tens of minutes $[9,10]$. Further increasing the charging speed usually results in an inferior performance and deteriorated material structure [11]. Ultrafast-charging SCs with charging rate $>10 \mathrm{~V} \mathrm{~s}^{-1}$ would significantly shorten the charging time and meet the requirements for high-rate energy storage devices [12-14]. Ultrafast-charging SCs are mainly fabricated by carbonaceous materials, such as activated carbon, graphene, and carbon nanotubes (CNTs) [15-18]. Yet, the relatively low electrical conductivity of activated carbon $\left(\sim 1\right.$ to $\left.100 \mathrm{~S} \mathrm{~m}^{-1}\right)$, chemically converted graphene $\left(\sim 500\right.$ to $\left.2000 \mathrm{~S} \mathrm{~m}^{-1}\right)$, and CNTs $\left(\sim 1 \times 10^{4} \mathrm{~S} \mathrm{~m}^{-1}\right)$ restricts their performances at ultrahigh charging rates $[19$, 20]. Therefore, exploration of new materials with superior conductivity for ultrafast-charging SCs is highly desirable.

Transition metal nitrides have received increasing attention for energy storage devices due to their excellent electrical conductivity and high capacities/capacitances [21, 22]. Among them, titanium nitride (TiN) stands out as one of the most promising material for SCs because of its outstanding conductivity $\left(4 \times 10^{5} \sim 5.55 \times 10^{6} \mathrm{~S} \mathrm{~m}^{-1}\right)$ and mechanical stability [22, 23]. However, in most cases, TiN nanostructures were supported on a substrate, which limits the gravimetric capacitances of TiN electrodes when it is normalized with the mass of entire electrode. Besides, most TiN-based electrodes suffered from severe capacitance loss in aqueous electrolytes, especially in the acidic and alkaline medium [24, 25]. Hasegawa et al. [25] showed that the neutral electrolyte can help to alleviate the cycling stability of transition metal nitrides. Here, we report the fabrication of a freestanding, flexible and porous TiN paper electrode for ultrafast-charging SCs. Electrical measurements showed that a single TiN nanobelt and a piece of TiN paper achieved excellent conductivities of $4.5 \times 10^{5}$ and $3.67 \times 10^{4} \mathrm{~S} \mathrm{~m}^{-1}$, respectively. The unique combination of high conductivity and pore structure of the TiN paper warrants rapid electron transport and ion diffusion that are required for ultrafast charging. The SC device also shows remarkable stability, which is uncommon for metal nitride materials.

\section{Materials and Methods}

\subsection{Synthesis of Ultralong $\mathrm{TiO}_{2}$ Nanobelts}

P25 powder $(0.1 \mathrm{~g})$ was mixed with $20 \mathrm{~mL} 10 \mathrm{~mol} \mathrm{~L}^{-1}$ $\mathrm{NaOH}$ aqueous solution. The mixture was transferred to a Teflon-lined autoclave and heated at $200{ }^{\circ} \mathrm{C}$ for $48 \mathrm{~h}$. The autoclave was cooled down at room temperature. The solid product, sodium titanate $\left(\mathrm{Na}_{2} \mathrm{Ti}_{3} \mathrm{O}_{7}\right)$ nanobelts, in the solution was collected by vacuum filtration and washed with deionized water. The sodium titanate was then re-dispersed into $0.1 \mathrm{~mol} \mathrm{~L}^{-1} \mathrm{HCl}$ aqueous solution and let it stay for $24 \mathrm{~h}$ to form hydrogen titanate $\left(\mathrm{H}_{2} \mathrm{Ti}_{3} \mathrm{O}_{7}\right)$ nanobelts through ion exchange reaction. Finally, $\mathrm{TiO}_{2}$ nanobelts were obtained by annealing the $\mathrm{H}_{2} \mathrm{Ti}_{3} \mathrm{O}_{7}$ nanobelts at $500{ }^{\circ} \mathrm{C}$ in air for $1 \mathrm{~h}$.

\subsection{Preparation of $\mathrm{TiO}_{2}$ and TiN Papers}

$\mathrm{TiO}_{2}$ and $\mathrm{TiN}$ paper were prepared by vacuum filtration of the ultralong $\mathrm{H}_{2} \mathrm{Ti}_{3} \mathrm{O}_{7}$ nanobelts followed by annealing process. First, the $\mathrm{H}_{2} \mathrm{Ti}_{3} \mathrm{O}_{7}$ nanobelts were re-dispersed into $100 \mathrm{~mL}$ deionized water and stirred for $0.5 \mathrm{~h}$ to make a uniform suspension. Then, the $\mathrm{H}_{2} \mathrm{Ti}_{3} \mathrm{O}_{7}$ nanobelt suspension solution was poured into the vacuum filtration system to get a $\mathrm{H}_{2} \mathrm{Ti}_{3} \mathrm{O}_{7}$ paper. The $\mathrm{H}_{2} \mathrm{Ti}_{3} \mathrm{O}_{7}$ paper with the filter paper was put in an electric oven at $70{ }^{\circ} \mathrm{C}$ for 20 min until they get dry. The $\mathrm{H}_{2} \mathrm{Ti}_{3} \mathrm{O}_{7}$ paper can be easily peeled off from the filter paper afterward. The mass loading of paper electrode can be readily adjusted by changing the amount of $\mathrm{H}_{2} \mathrm{Ti}_{3} \mathrm{O}_{7}$ nanobelt suspension in the solution for the filtration.

$\mathrm{TiO}_{2}$ paper was obtained by annealing the $\mathrm{H}_{2} \mathrm{Ti}_{3} \mathrm{O}_{7}$ paper at $500{ }^{\circ} \mathrm{C}$ in air for $1 \mathrm{~h}$. TiN papers were obtained by annealing the $\mathrm{TiO}_{2}$ paper in ammonia environment at $800{ }^{\circ} \mathrm{C}$ for $1 \mathrm{~h}$. The conventional TiN pellet electrodes as a control sample were fabricated by mixing the TiN nanobelts, carbon black, and PTFE in a ratio of 8:1:1 followed by rolling the mixture into thin films.

\subsection{Materials Characterization}

The X-ray diffraction (XRD) patterns were collected on a powder X-ray diffractometer (Rigaku Americas Miniflex 
Plus) with $2 \theta$ angle from $30^{\circ}$ to $70^{\circ}$ under a step size of $0.01^{\circ}$ at a rate of $1^{\circ} \mathrm{min}^{-1}$. The morphology of nanobelts was investigated by a field emission scanning electron microscopy (SEM, FEI Quanta 3D FEG dual beam) and transmission electron microscopy (TEM, JEM, 2010-HR). X-ray photoelectron spectroscopy (XPS, ESCALAB 250) was used to analyze the chemical composition of samples. Textural properties were examined by Brunauer-Emmett-Teller and Barrett-Joyner-Halenda methods using an ASAP 2020 surface area analyzer (Micromeritics Instrument) via nitrogen porosimetry. The areal mass of the electrodes was measured based on $4 \mathrm{~cm}^{2}$ TiN papers on an analytical balance (Citizen CX265) with a resolution of $0.01 \mathrm{mg}$. The thickness of the electrodes was measured using a micrometer caliper (NSCING) with a resolution of $0.001 \mathrm{~mm}$. The single $\mathrm{TiO}_{2}$ and TiN nanobelt devices were fabricated via a focused ion beam (FIB, Quanta 3D FEG) with Pt as the contact electrode. The electrical measurement was carried out using an Agilent 2400 instrument.

The electrochemical measurements were conducted using electrochemical workstation (CHI 660D and ECLab SP-300). For three-electrode measurements, a piece of $0.2 \mathrm{~cm}^{2} \mathrm{TiN}$ paper was used as working electrode. $\mathrm{Ag} / \mathrm{AgCl}$ (CHI, USA) and YP-50 activated carbon (Kuraray Chemical, Japan) were used as the reference electrode and counter electrode, respectively. The measurements were carried out in different aqueous electrolytes, including $0.5 \mathrm{M} \mathrm{Na}_{2} \mathrm{SO}_{4}$ (pH 7.67), $3 \mathrm{M} \mathrm{LiCl}$ (pH 6.76), $1 \mathrm{M} \mathrm{H}_{2} \mathrm{SO}_{4}$ (pH 0.03), and $1 \mathrm{M} \mathrm{KOH}$ ( $\mathrm{pH} 13.65$ ) solutions. A piece of Celgard film was used as a separator (Celgard, USA). Two-electrode symmetric devices were assembled with two pieces of $0.2 \mathrm{~cm}^{2}$ TiN paper or pellet with the same area and mass loading $\left(\sim 1.5 \mathrm{mg} \mathrm{cm}^{-2}\right)$. Three samples with similar mass loadings were tested for each condition to make sure the electrode's capacitive performance is reproducible.

\subsection{Calculation}

Gravimetric capacitance is calculated from CV curves using Eq. 1:

$C_{\mathrm{g}}=\frac{\int I \mathrm{~d} V}{v \Delta V m}$

where $I$ is the current (A), $V$ is the working potential, $v$ is the scan rate $\left(\mathrm{V} \mathrm{s}^{-1}\right), \Delta V$ is the working voltage, $m$ is the mass loading (g). $\int I \mathrm{~d} V$ corresponds to the area of the discharging parts. For the working potential in the positive region, it corresponds the area in the reductive part. For the working potential in the negative region, it corresponds to the area in the oxidation part.

Areal capacitance is calculated from CV curves using Eq. 2

$C_{A}=\frac{\int I \mathrm{~d} V}{v \Delta V A}$

where $I$ is the current (A), $V$ is the working potential, $\mathrm{v}$ is the scan rate $\left(\mathrm{V} \mathrm{s}^{-1}\right), \Delta V$ is the working voltage, $A$ is the working area $\left(\mathrm{cm}^{2}\right) . \int I \mathrm{~d} V$ corresponds to the area of the discharging parts.

The characteristic time constant $\left(\tau_{\text {characteristic }}\right)$ is calculated by Eq. 3:

$\tau_{\text {characteristic }}=\frac{1}{2 \pi f_{\text {characteristic }}}$

where the $f_{\text {characteristic }}$ is the frequency $(\mathrm{Hz})$ at a phase degree of $-45^{\circ}$ from the EIS measurement.

Imaginary capacitances $\left(C^{\prime \prime}\right)$ were calculated by Eq. 4 :

$C^{\prime \prime}=\frac{Z^{\prime}}{2 \pi f|Z|^{2}}$

where $Z^{\prime}(\Omega)$ is the real part of $Z, Z$ is the electrochemical impedance $(\Omega), f$ is the frequency $(\mathrm{Hz})$ from the EIS measurement.

\section{Results and Discussions}

Ultralong $\mathrm{TiO}_{2}$ nanobelts were prepared by a hydrothermal method. $\mathrm{TiO}_{2}$ nanobelts have average length around tens of micrometers, width of 50-200 nm and thickness of 20-50 nm (Fig. S1). These nanobelts with large aspect ratio can be easily assembled into flexible, paper-like electrodes using filtration method (Fig. S2) [26]. XRD patterns confirmed that the nanobelts are monoclinic $\mathrm{TiO}_{2}\left(\mathrm{TiO}_{2}-\mathrm{B}\right.$, JCPDS No. 74-1940) (Fig. S3). TiN paper was obtained by treating the $\mathrm{TiO}_{2}$ paper in ammonia atmosphere at $800{ }^{\circ} \mathrm{C}$ (Fig. 1a). Notably, TiN paper inherits the excellent flexibility of $\mathrm{TiO}_{2}$ paper (Fig. 1b inset). While the basic framework of the 3D nanobelts assembly did not change upon ammonia treatment, each nanobelt became porous structure (Fig. 1b, c). $\mathrm{N}_{2}$ adsorption-desorption isotherms showed that the specific surface area increased from $31.2 \mathrm{~m}^{2} \mathrm{~g}^{-1}\left(\mathrm{TiO}_{2}\right.$ paper) to $43.5 \mathrm{~m}^{2} \mathrm{~g}^{-1}$ (TiN paper) after ammonia treatment (Fig. S4). The hysteresis located at $0.4<\mathrm{P} / \mathrm{P}_{0}<1.0$ indicates 


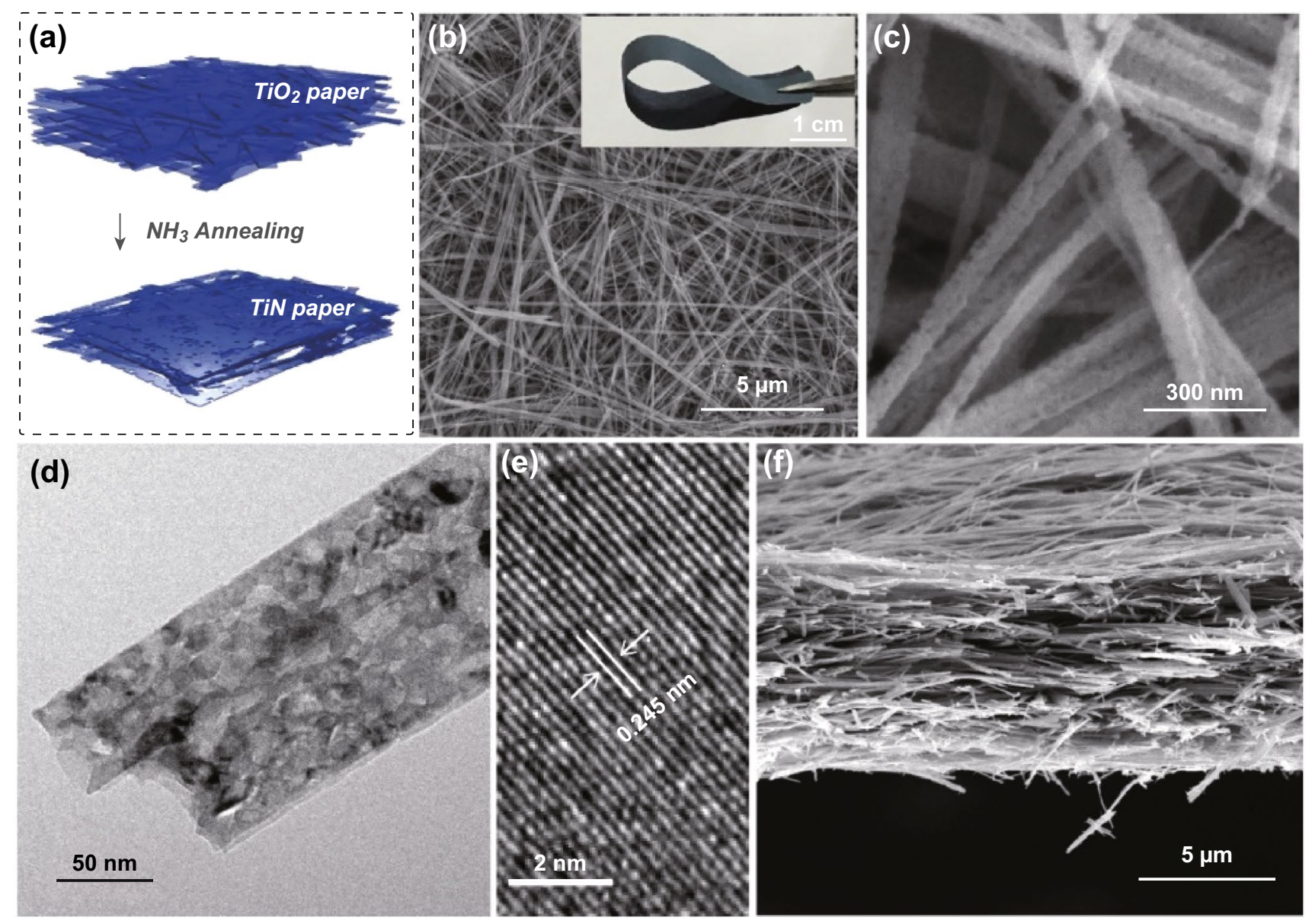

Fig. 1 a Schematic illustration of the fabrication process of TiN paper from $\mathrm{TiO}_{2}$ paper. $\mathbf{b}, \mathbf{c} \mathrm{SEM}$ images of TiN nanobelts. Inset in $\mathbf{b}$ shows the digital image of a piece of flexible TiN paper. d, e TEM and high-resolution TEM images of porous TiN nanobelts. f Cross-sectional SEM image of TiN paper

the presence of mesopores [27]. The pore size distribution profile also confirmed the presence of higher amount of mesopores for TiN paper (Fig. S5). The porous structure was further confirmed with TEM image (Fig. 1d). It has been reported that $\mathrm{TiO}_{2}$ can be etched by ammonia at high temperatures $[21,22]$. The topotactic reaction of $\mathrm{TiO}_{2}$ nanobelts and $\mathrm{NH}_{3}$ led to the rearrangement of the oxide structure and produced a mesoporous structure in the framework of the TiN nanobelts [28, 29]. The high-resolution TEM image revealed the TiN nanobelt has a lattice fringe of $0.245 \mathrm{~nm}$, which is consistent with the $d$-spacing of (111) crystal plane of cubic TiN (Fig. 1e). The cross-sectional SEM image clearly showed that the TiN paper is formed via uniform assembly of nanobelts (Fig. 1f). The filtration method allows good control of the TiN paper thickness. The large pores between the nanobelts and mesoporous structure of the nanobelts provide sufficient space for ion diffusion, which is critical for the fast charging.

Additional X-ray diffraction and spectroscopy techniques were used to probe the chemical composition of the TiN paper. Despite XRD pattern showed that the sample is cubic phase TiN (JCPDS No. 38-1420) (Fig. 2a), XPS survey spectrum revealed the existence of $\mathrm{Ti}, \mathrm{N}$, and $\mathrm{O}$ on the surface of TiN nanobelts (Fig. S6). The O signal is believed to be due to $\mathrm{TiO}_{2}$ and/or $\mathrm{TiO}_{x} \mathrm{~N}_{y}$, which might come from the incomplete conversion from their oxide predecessor and surface oxidation after exposure in air $[25,30]$. The possible reactions between $\mathrm{TiO}_{2}$ and $\mathrm{NH}_{3}$ are ammonia first decomposes into nitrogen and hydrogen gas $\left(2 \mathrm{NH}_{3}+\right.$ heat $\left.\rightarrow \mathrm{N}_{2}+3 \mathrm{H}_{2}\right)$. The reaction proceeds with the reduction of $\mathrm{TiO}_{2}$ to $\mathrm{TiO}$ by hydrogen gas $\left(\mathrm{TiO}_{2}+\mathrm{H}_{2} \rightarrow \mathrm{TiO}+\mathrm{H}_{2} \mathrm{O}\right)$. Then, TiO reacts with 
ammonia and generate $\mathrm{TiN}\left(\mathrm{TiO}+\mathrm{NH}_{3} \rightarrow \mathrm{TiN}+\mathrm{H}_{2} \mathrm{O}\right)$ [31]. The $\mathrm{TiO}_{2}$ can also react with nitrogen and generate $\mathrm{TiO}_{x} \mathrm{~N}_{y}\left(\mathrm{TiO}_{2}+\mathrm{N}_{2} \rightarrow \mathrm{TiO}_{\mathrm{x}} \mathrm{N}_{\mathrm{y}}+\mathrm{O}_{2}\right)$ [32].

The core-level Ti $2 p$ XPS spectrum exhibits multiple peaks in the binding energy range between 453 and $466 \mathrm{eV}$. They can be deconvoluted into three sets of synthetic peaks, corresponding to Ti-N $\left(2 \mathrm{p}_{3 / 2}=455.4 \mathrm{eV}, 2 \mathrm{p}_{1 / 2}=461.3 \mathrm{eV}\right)$, $\mathrm{Ti}-\mathrm{N}-\mathrm{O}\left(2 \mathrm{p}_{3 / 2}=456.85 \mathrm{eV}, 2 \mathrm{p}_{1 / 2}=462.9 \mathrm{eV}\right)$ and Ti-O $\left(2 \mathrm{p}_{3 / 2}=458.4 \mathrm{eV}, 2 \mathrm{p}_{1 / 2}=464.15 \mathrm{eV}\right)$ (Fig. 2b) [33] The $\mathrm{N}$ 1s spectrum also consists of two different peaks, Ti-N $(396.6 \mathrm{eV})$ and $\mathrm{Ti}-\mathrm{N}-\mathrm{O}(398.6 \mathrm{eV})$, which are consistent with the peaks observed in the Ti $2 p$ spectrum (Fig. 2c) [34] Furthermore, TEM elemental mapping results confirmed the uniform distribution of $\mathrm{Ti}, \mathrm{N}$, and $\mathrm{O}$ in $\mathrm{TiN}$ nanobelts (Fig. 2d).

To probe the electrical conductivity of $\mathrm{TiO}_{2}$ and $\mathrm{TiN}$, focused ion beam (FIB) lithography was used to fabricate single-nanobelt devices (Fig. 3a inset). The current-voltage $(I-V)$ curves of the $\mathrm{TiO}_{2}$ and $\mathrm{TiN}$ nanobelt devices are shown in Fig. 3a. TiN exhibits a significantly larger current response with voltage than $\mathrm{TiO}_{2}$. Their specific conductivities were calculated according to Eqs. 5 and 6 :
$R=\rho \frac{l}{A}$

$\sigma=\frac{1}{\rho}$

where $R$ is the resistance, $\rho$ is the resistivity, $l$ is the length of the nanobelt, $A$ is the cross-sectional area of the nanobelt, and $\sigma$ is the conductivity of the nanobelt. The conductivity of a single TiN nanobelt $\left(4.5 \times 10^{5} \mathrm{~S} \mathrm{~m}^{-1}\right)$ is almost 3 orders of magnitude higher than that of $\mathrm{TiO}_{2}$ nanobelt $\left(4.9 \times 10^{2} \mathrm{~S} \mathrm{~m}^{-1}\right)$. The TiN paper also retains excellent conductivity of $3.67 \times 10^{4} \mathrm{~S} \mathrm{~m}^{-1}$. These values are much higher than the previous reported carbon-based materials, such as activated carbon (10-100 $\left.\mathrm{S} \mathrm{m}^{-1}\right)$ [35], chemical converted graphene $\left(5 \times 10^{2} \mathrm{~S} \mathrm{~m}^{-1}\right)$ [20], holy graphene $\left(\sim 10^{3} \mathrm{~S} \mathrm{~m}^{-1}\right)$ [36], laser-scribed graphene $\left(1738 \mathrm{~S} \mathrm{~m}^{-1}\right)$ and even higher than the commercial CNT $\left(\sim 10^{4} \mathrm{~S} \mathrm{~m}^{-1}\right)$ [37].

Furthermore, the TiN paper has a low sheet resistance of only $2.73 \Omega \mathrm{sq}^{-1}$, which is smaller than those of graphene film $\left(280 \Omega \mathrm{sq}^{-1}\right)$ [38], chemical converted graphene film (124 $\left.\Omega \mathrm{sq}^{-1}\right)$ [39], CNT paper $\left(10 \Omega \mathrm{sq}^{-1}\right)$ [40], Au paper $\left(7 \Omega \mathrm{sq}^{-1}\right)$ [41], $\mathrm{MoO}_{3-x}$ paper $\left(5.1 \Omega \mathrm{sq}^{-1}\right)$ [37], Polypyrrole paper $\left(4.5 \Omega \mathrm{sq}^{-1}\right)$ [9], and comparable to that for
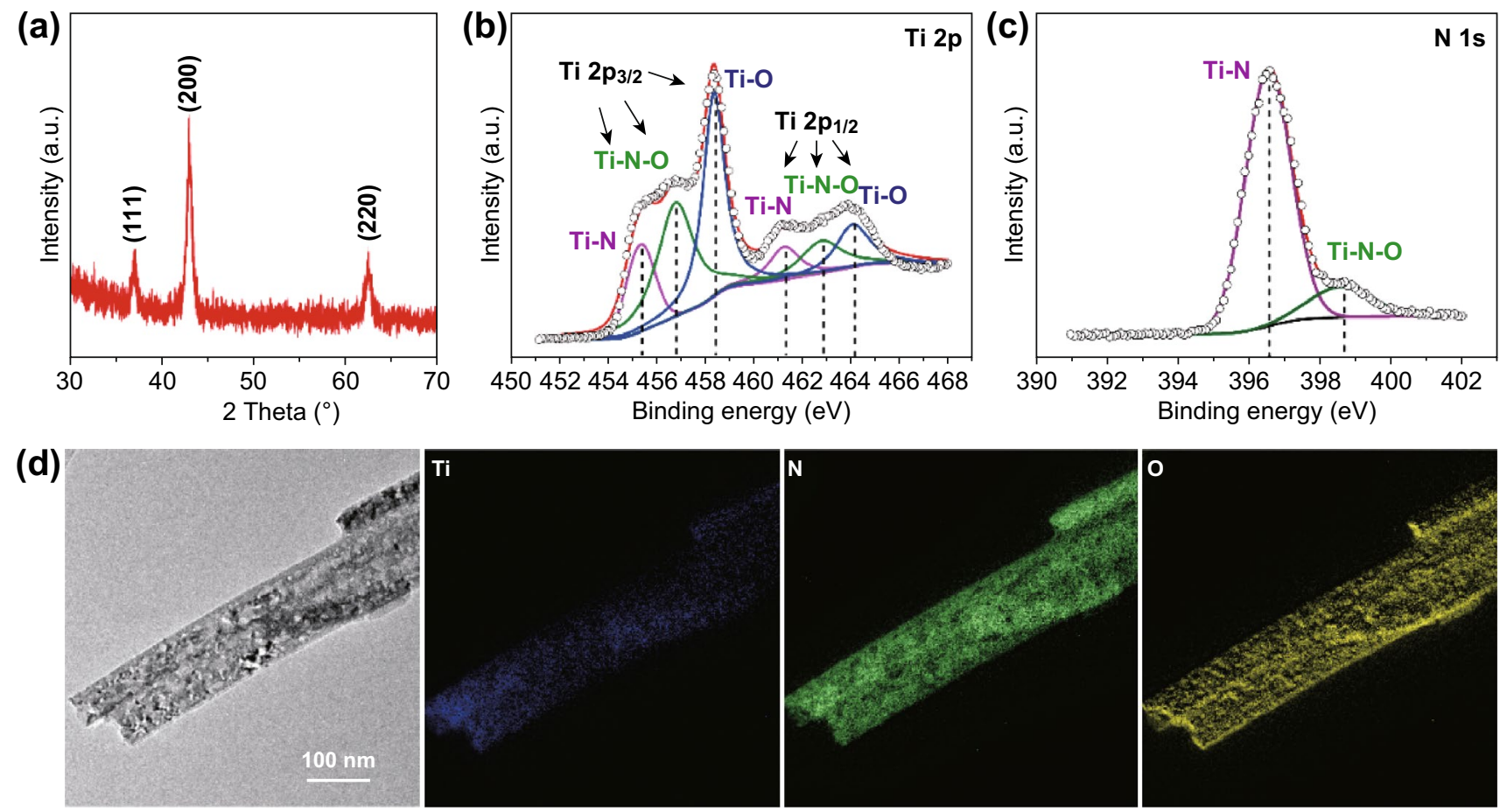

Fig. 2 a XRD pattern and b, c Ti $2 p$ and $N$ 1s XPS spectra of TiN paper. d TEM image and the corresponding Ti, N, and O elemental mapping images of TiN nanobelts 

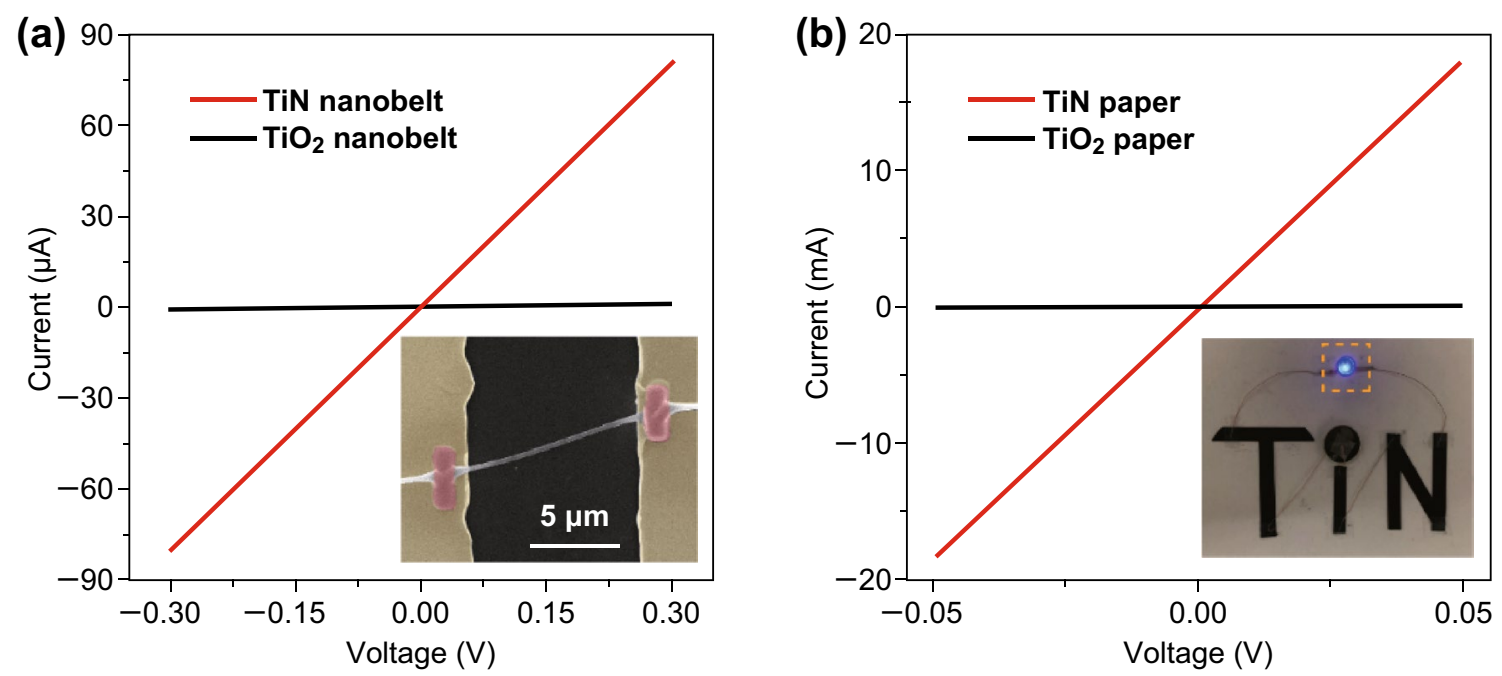

Fig. 3 a $I-V$ curves of individual ultralong $\mathrm{TiO}_{2}$ and TiN nanobelts. Inset shows the SEM image of a single nanobelt test unit. b $I-V$ curves of $\mathrm{TiO}_{2}$ and TiN papers. Inset shows the digital image of TiN papers as a part of the electrical connections to light a blue LED by a commercial $3 \mathrm{~V}$ battery. (Color figure online)

poly(3,4-ethylenedioxythiophene):polystyrene sulfonate (PEDOT:PSS) paper (2.60 $\Omega \mathrm{sq}^{-1}$ ) [42] (Fig. 3b). Given the excellent conductivity, the TiN paper can actually be used as a connecting lead to power a $2.5 \mathrm{~V}$ light-emitting-diode (LED) with a commercial $3 \mathrm{~V}$ button battery (Figs. $3 b$ inset and S7).

The unique combination of high conductivity and pore structure makes TiN paper an excellent electrode candidate for ultrafast charging supercapacitors. The working potential of TiN papers was first evaluated using a three-electrode system in electrolytes with different $\mathrm{pH}$ values. TiN paper electrodes exhibit the largest working potential window $(1.5 \mathrm{~V})$ in $0.5 \mathrm{M} \mathrm{Na}_{2} \mathrm{SO}_{4}$ (pH 7.57) neutral electrolyte, while only $0.8 \mathrm{~V}$ in $1 \mathrm{M} \mathrm{KOH}(\mathrm{pH} 13.65), 1 \mathrm{~V}$ in $1 \mathrm{M} \mathrm{H}_{2} \mathrm{SO}_{4}(\mathrm{pH} 0.03$ ), and $0.9 \mathrm{~V}$ in $3 \mathrm{M} \mathrm{LiCl}$ (pH 6.76) (Fig. S8). Significantly, this is the first report for TiN-based materials to be operated in such a wide working voltage of $1.5 \mathrm{~V}$ [22, 33, 43-46]. The large working voltage can be ascribed to the employment of the sulfate-based neutral electrolyte, which have been demonstrated to be effective in expanding the working voltage of SC materials in aqueous electrolyte because the high solvation energy of sulfate and alkali metal ions (160-220 kJ mol${ }^{-1}$ ) cause relatively large overpotentials for hydrogen evolution and oxygen evolution reactions [47-50].

Excellent specific capacitances have been obtained for electrodes with small mass loading $\left(0.1-1 \mathrm{mg} \mathrm{cm}^{-2}\right)$ of metal nitrides deposited on conducting substrates $(\sim 10$ to
$200 \mathrm{mg} \mathrm{cm}^{-2}$ ) [21, 22, 24, 33, 51]. These capacitances were typically calculated based only on the mass of active material. However, the value of specific capacitance would be more practically meaningful if it is normalized to the mass of the entire electrode. In this regard, binder-free and conducting additive-free TiN papers are advantageous over its counterparts that require current collector. The TiN paper electrode showed a high capacitance of $164.5 \mathrm{~F} \mathrm{~g}^{-1}$ in $0.5 \mathrm{M}$ $\mathrm{Na}_{2} \mathrm{SO}_{4}$ at a scan rate of $5 \mathrm{mV} \mathrm{s}^{-1}$ and retained $64.7 \%$ of its capacitance when the scan rate is raised to $100 \mathrm{mV} \mathrm{s}^{-1}$ (Fig. S9), which is significantly higher than capacitance of other metal nitride electrodes normalized to the mass of entire electrode, such as TiN nanosheets/graphene nanosheets $\left(5.3 \mathrm{~F} \mathrm{~g}^{-1}\right.$ at $\left.10 \mathrm{mV} \mathrm{s}^{-1}\right)$ [21], $\mathrm{Nb}_{4} \mathrm{~N}_{5} / \mathrm{Ni}$ foil $\left(0.86 \mathrm{~F} \mathrm{~g}^{-1}\right.$ at $\left.0.67 \mathrm{~A} \mathrm{~g}^{-1}\right)$ [52], TiN nanowire/carbon cloth $\left(10.2 \mathrm{~F} \mathrm{~g}^{-1}\right.$ at $\left.10 \mathrm{mV} \mathrm{s}^{-1}\right)$ [22], $\mathrm{VN}$ nanowire/carbon cloth (16.7 $\mathrm{F} \mathrm{g}^{-1}$ at $10 \mathrm{mV} \mathrm{s}^{-1}$ ) [53], and $\mathrm{TiN} / \mathrm{MnO}_{2}$ nanowire/ carbon cloth $\left(25.9 \mathrm{~F} \mathrm{~g}^{-1}\right.$ at $\left.2 \mathrm{~mA} \mathrm{~cm}^{-2}\right)$ [54].

TiN pellet electrodes as control samples were prepared by mixing TiN nanobelts, carbon black and PTFE binders, followed by pressing the mixture into thin pellets (Figs. 4a and S10). Symmetric supercapacitors (SSCs) were prepared via the assembly of two TiN paper or pellet electrodes with the same mass loadings. As shown in Fig. 4b, the paperbased SSCs indeed have considerably longer charging and discharging time than the pellet SSCs. Importantly, the paper SSC exhibits excellent capacitive behavior even at ultrafast 
(a)
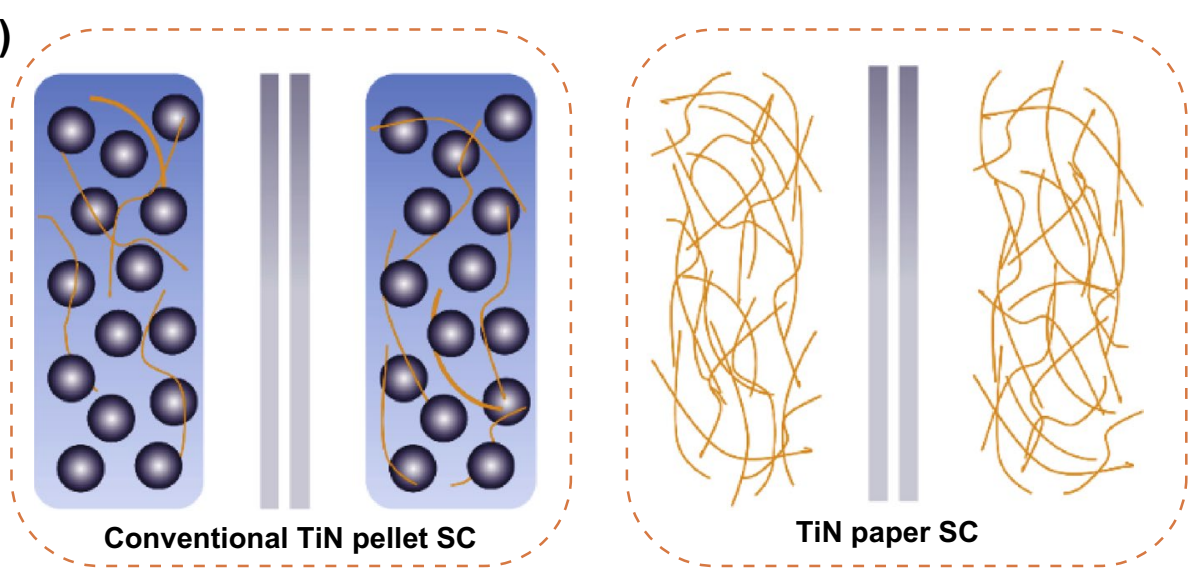

Binder (PTFE)

Activated carbon

Separator

TiN nanobelts
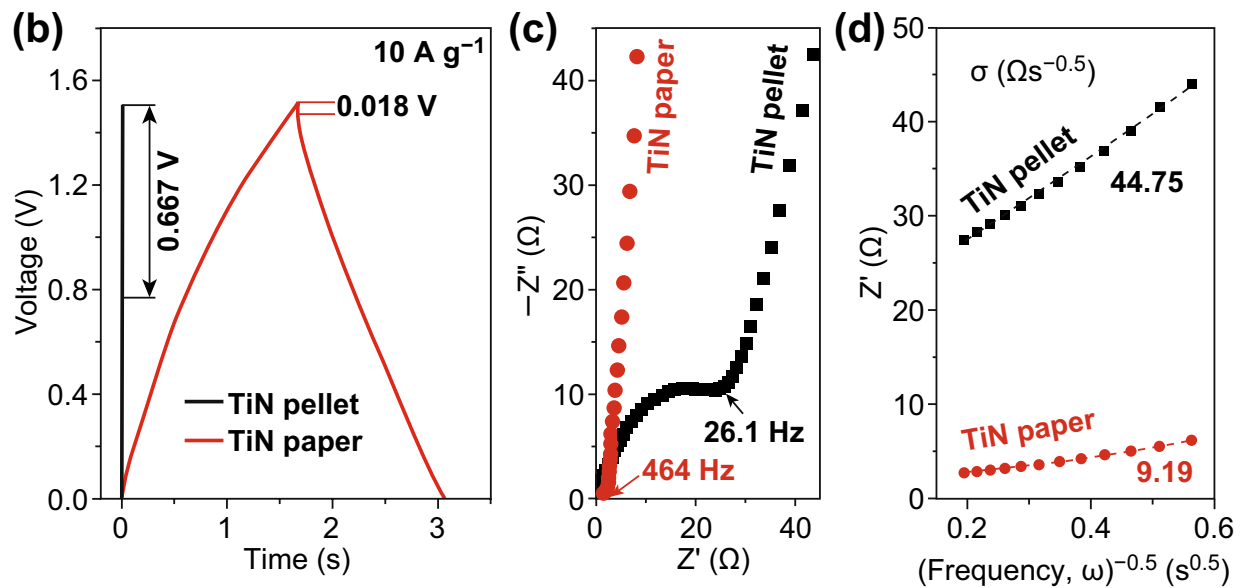

Fig. 4 a Schematic illustration of the device configuration of TiN pellet and paper-based SSCs. b Galvanostatic charging and discharging curves of the SSCs at a current density of $10 \mathrm{~A} \mathrm{~g} \mathrm{~g}^{-1}$. The iR drop of TiN paper SSC and pellet SSC are 0.018 and $0.667 \mathrm{~V}$. c Electrochemical impedance spectra of TiN pellet SSC and TiN paper SSC. The knee frequency of the TiN paper SSCs and TiN pellet SSCs are 464 and $26.1 \mathrm{~Hz}$. d Z' versus the reciprocal of the square root of frequency $\left(\omega^{-0.5}\right)$ in the intermediate frequency range. The dashed lines are the linear fitting lines for calculating the ion diffusion resistance, $\sigma$

charging rates at $200 \mathrm{~A} \mathrm{~g}^{-1}$ (Fig. S11), while the charging rate of the pellet SSC is limited by its large internal resistances (Fig. S12). As shown in Fig. S13, the TiN paper-based SSC achieves a specific capacitance of $12.67 \mathrm{~F} \mathrm{~g}^{-1}$ at a scan rate of $100 \mathrm{mV} \mathrm{s}^{-1}$ and retains a capacitance of $8.99 \mathrm{~F} \mathrm{~g}^{-1}$ at a high scan rate of $1 \mathrm{~V} \mathrm{~s}^{-1}$ and $3.35 \mathrm{~F} \mathrm{~g}^{-1}$ at an ultrahigh scan rate of $100 \mathrm{~V} \mathrm{~s}^{-1}$. These values are substantially higher than that of pellet-based SSC. The reduced capacitance and rate capability of the pellet electrode are mainly because of two reasons. First, the addition of non-conductive and non-electrochemical active polymer binder (PTFE) increases the overall electrode resistance and decreases the specific capacitance. Second, part of the active capacitive material
TiN nanobelts is covered by carbon black and PTFE. This makes the ion diffusion to TiN nanobelts more difficult, especially at high charging rates, compared to the porous paper electrode.

EIS measurements were performed to understand the electron transport, ion diffusion resistivity and frequency characteristics of TiN SSCs. The equivalent series resistance (ESR) obtained from the intercept of the plot on the real axis is only $0.92 \Omega$, indicating the excellent electrical conductivity and low resistance of TiN paper SSCs (Fig. S14). Besides, the paper SSC showed much smaller charge transfer resistance $(1.66 \Omega)$ than the pellet SSC (35.98 $\Omega$ ) (Fig. 4c). The high knee frequency of paper SSC $(464 \mathrm{~Hz})$ is also an order of 
magnitude higher than the knee frequency of the pellet SSC $(26.1 \mathrm{~Hz})$ (Fig. 4c). The characteristic time constant of the paper SSC was calculated to be $4 \mathrm{~ms}$ (Fig. S15). This value is substantially smaller than the values reported for many carbon-based SSCs, including CNT fibers (1930 ms) [55], metal-organic frameworks derived porous carbon (1270 ms) [56], carbide-derived carbon (379 ms) [57], laser-scribed graphene (33 ms) [58], and onion-like carbon (26 ms) [59].

In addition to the efficient electron transport, paper SSCs exhibited much faster ion diffusion kinetics than the conventional pellet SSCs. The ion diffusion resistances $(\sigma)$ can be extracted from the slopes of the linear fitting of the real part of impedance $\left(Z^{\prime}\right)$ versus the reciprocal of the square root of frequency $\left(\omega^{-0.5}\right)$ in the intermediate frequency range [60]. The paper SSC displayed an $\sigma$ of $9.19 \Omega \mathrm{s}^{-0.5}$, which is much smaller than the pellet SSCs $\left(44.75 \Omega \mathrm{s}^{-0.5}\right)$, highlighting the advantage of having the unique porous electrode structure (Fig. 4d).

The CV curves of the paper SSC retain the rectangular shape even at ultrafast charging rates of $100 \mathrm{~V} \mathrm{~s}^{-1}$ (Fig. 5a-e). The linear increase of the discharge currents to $20 \mathrm{~V} \mathrm{~s}^{-1}$ reflects the efficient charge transfer and ion diffusion in the paper SSCs (Fig. 5f), in contrast to the pellet SSCs. The paper SCC delivers an energy density of 1.05 Wh $\mathrm{kg}^{-1}$ under an extraordinarily high power density of $251.2 \mathrm{~kW} \mathrm{~kg}^{-1}$, with a charging/discharging time of only $15 \mathrm{~ms}$. These values are much better than most of previously reported electrochemical capacitors (Fig. S16). Furthermore, the TiN paper SSCs showed remarkable energy density of $3.26 \mathrm{mWh} \mathrm{cm}^{-3}$ under a power density of 78.3 $\mathrm{mW} \mathrm{cm}{ }^{-3}$. An energy density of $0.54 \mathrm{mWh} \mathrm{cm}^{-3}$ was still retained under an extremely high power density of $130,632.2 \mathrm{~mW} \mathrm{~cm}^{-3}$, which is again much higher than most metal nitride-based SSCs (Fig. S17) [21, 22, 51, 53, 61, 62].

Transition metal nitride electrodes have been suffering from the instability problem during cycling [22, 24]. TiN paper SSCs were tested for long-term stability in three different electrolytes, $0.5 \mathrm{M} \mathrm{Na}_{2} \mathrm{SO}_{4}, 1 \mathrm{M} \mathrm{H}_{2} \mathrm{SO}_{4}$, and $1 \mathrm{M} \mathrm{KOH}$ solutions. TiN paper SSC shows zero decay in capacitance after cycling in $0.5 \mathrm{M} \mathrm{Na}_{2} \mathrm{SO}_{4}$ electrolyte for 200,000 cycles at $1 \mathrm{~V} \mathrm{~s}^{-1}$, while only $47.5 \%$ and $42.4 \%$ of capacitance were retained in $1 \mathrm{M} \mathrm{H}_{2} \mathrm{SO}_{4}$ and
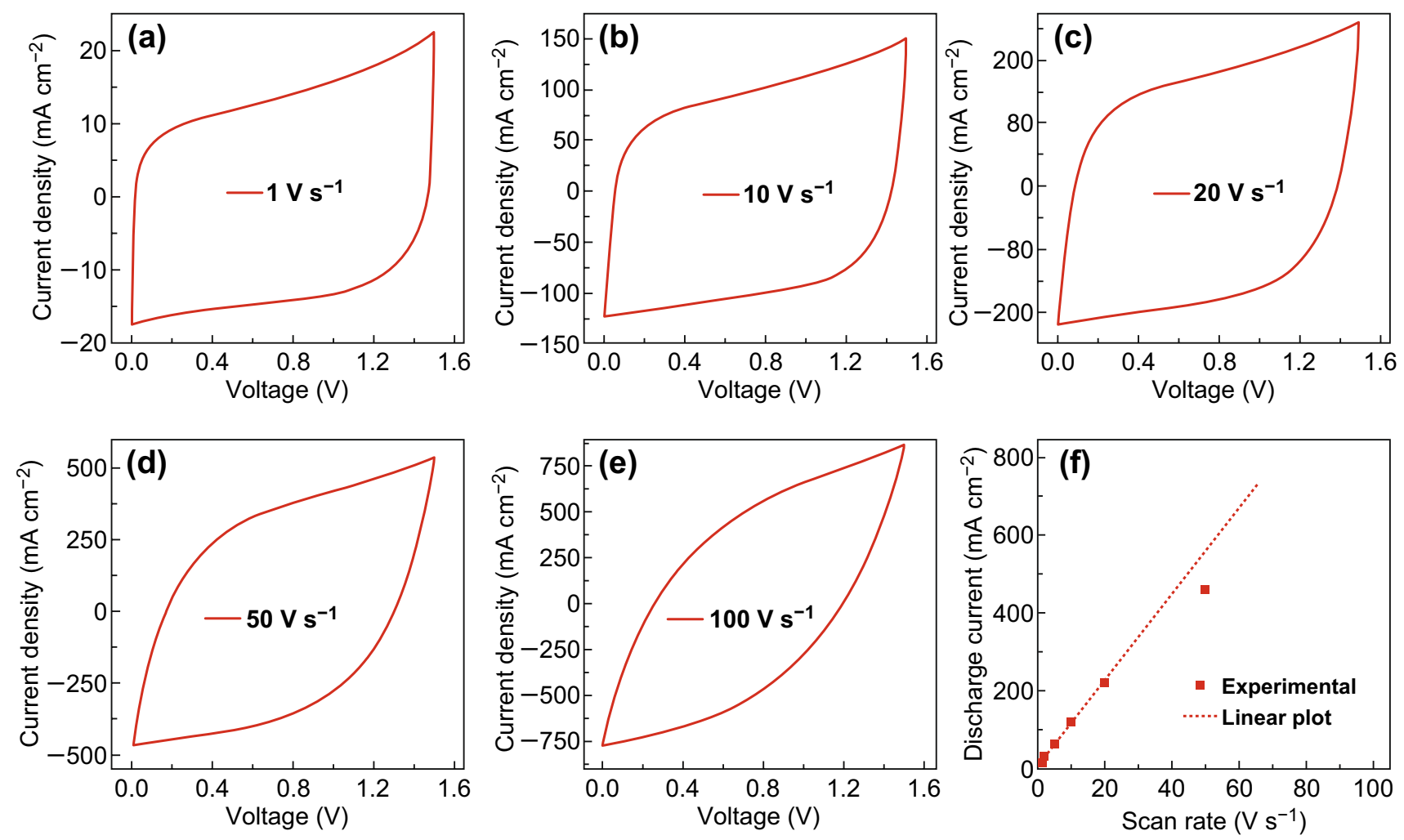

Fig. 5 a-e CV curves of TiN paper SSC collected at different scan rates in $0.5 \mathrm{M} \mathrm{Na}_{2} \mathrm{SO}_{4}$ aqueous electrolyte. f A plot of discharge current of TiN paper SSC against scan rate 

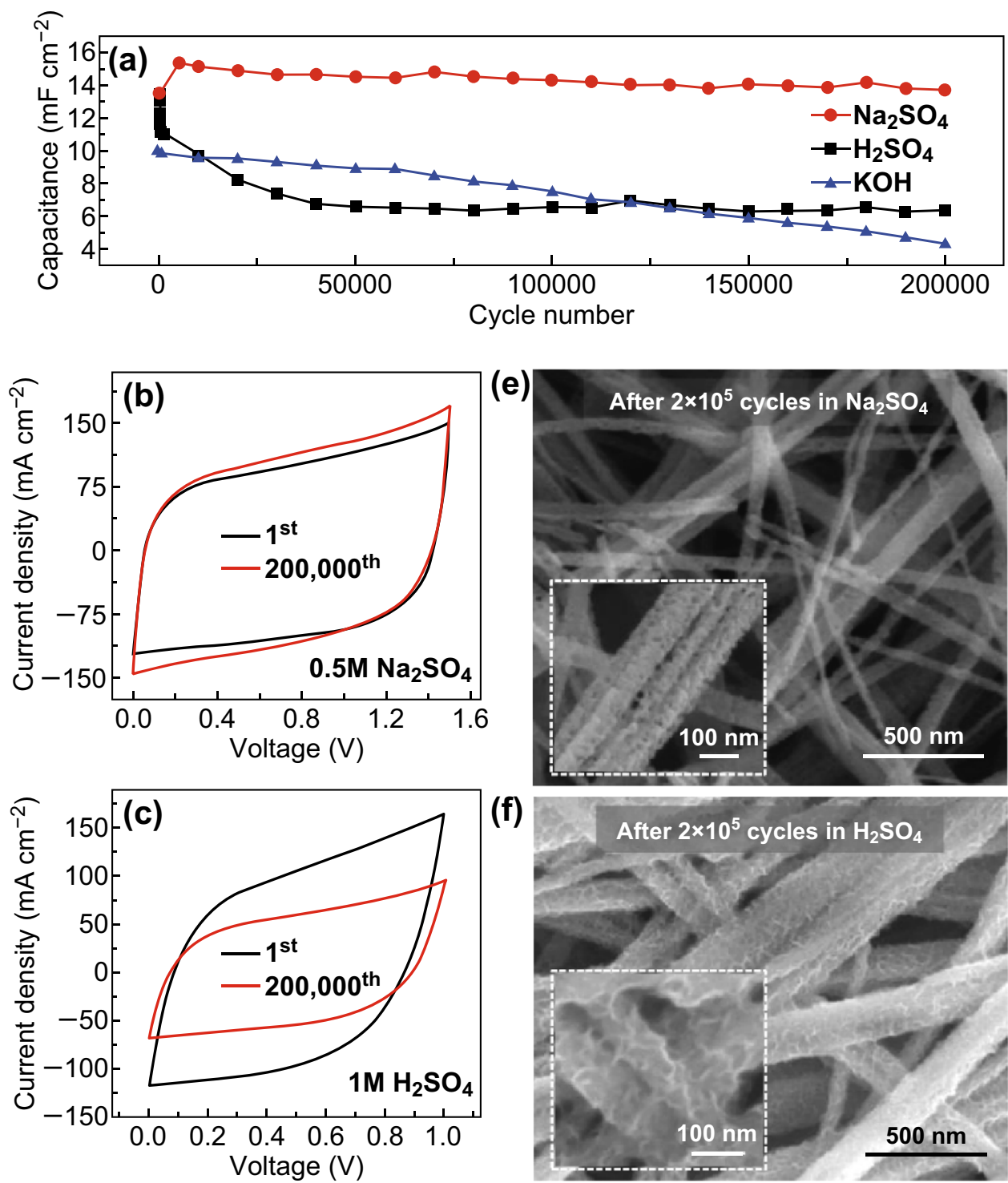

(f)
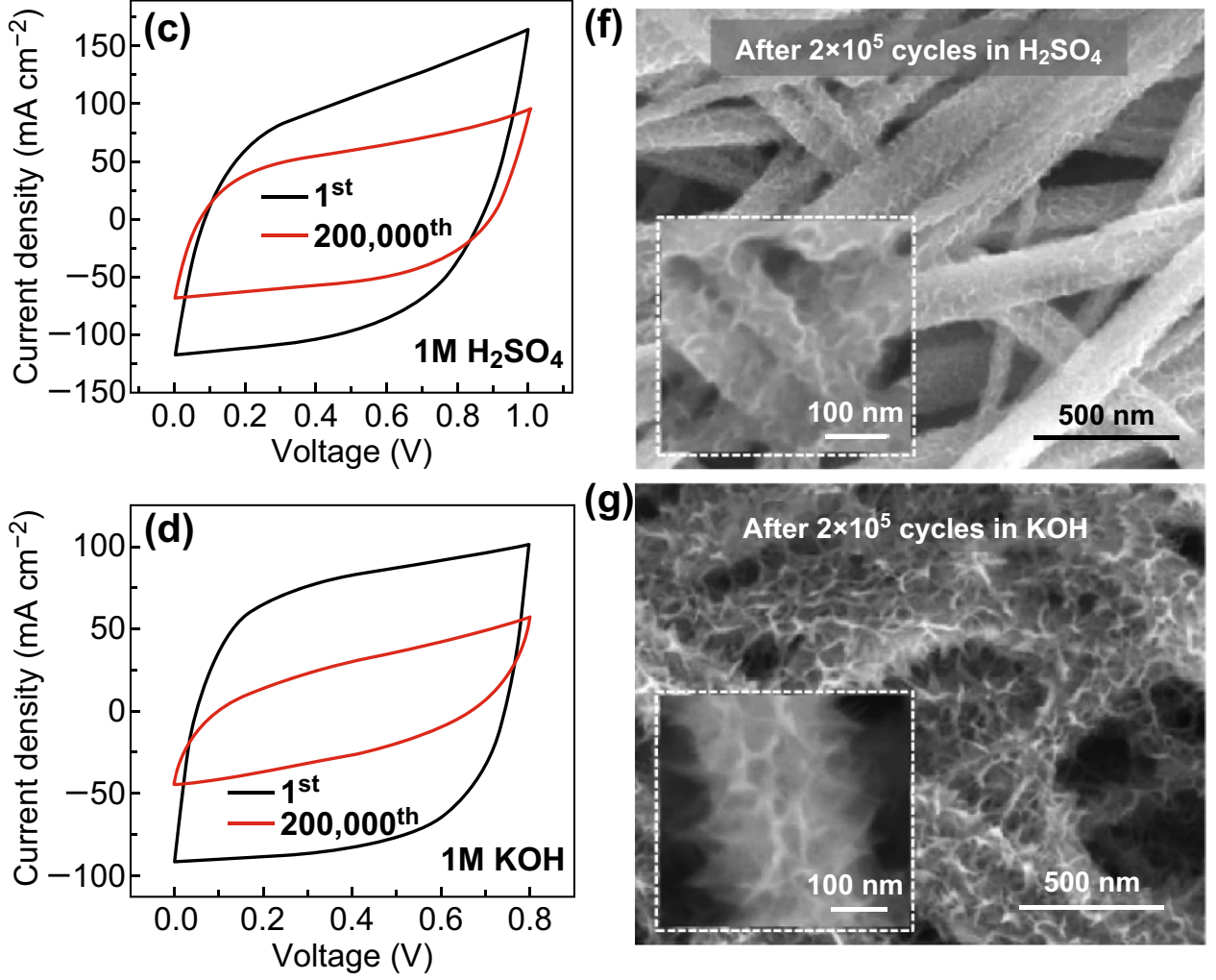

(g)

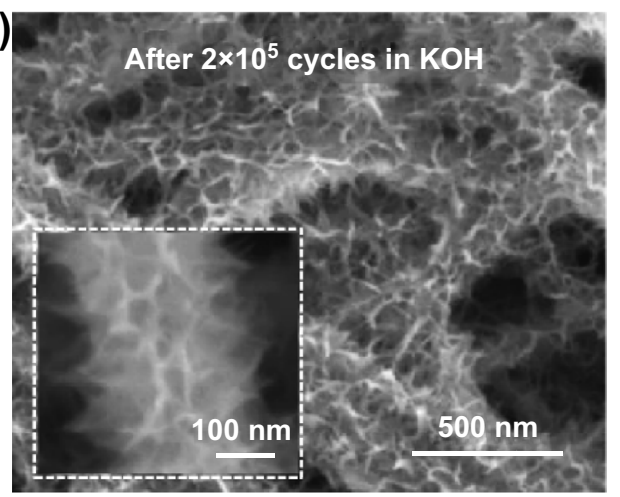

Fig. 6 a Capacitance retention of TiN paper SSC obtained at $1 \mathrm{~V} \mathrm{~s}^{-1}$ in $0.5 \mathrm{M} \mathrm{Na}_{2} \mathrm{SO}_{4}, 1 \mathrm{M} \mathrm{H}_{2} \mathrm{SO}_{4}$, and $1 \mathrm{M} \mathrm{KOH}$ electrolytes are plotted as a function of cycling number. b-d CV curves of TiN paper SSC collected at the 1st and after the 200,000th cycle in $0.5 \mathrm{M} \mathrm{Na}_{2} \mathrm{SO}_{4}, 1 \mathrm{M} \mathrm{H}_{2} \mathrm{SO}_{4}$, and $1 \mathrm{M} \mathrm{KOH}$ electrolytes at $10 \mathrm{~V} \mathrm{~s}^{-1}$. e-g SEM images of the TiN paper electrodes after testing for 200,000 cycles in $0.5 \mathrm{M} \mathrm{Na}_{2} \mathrm{SO}_{4}, 1 \mathrm{M}^{-}$ $\mathrm{H}_{2} \mathrm{SO}_{4}$, and $1 \mathrm{M} \mathrm{KOH}$ electrolytes at $1 \mathrm{~V} \mathrm{~s}^{-1}$ 

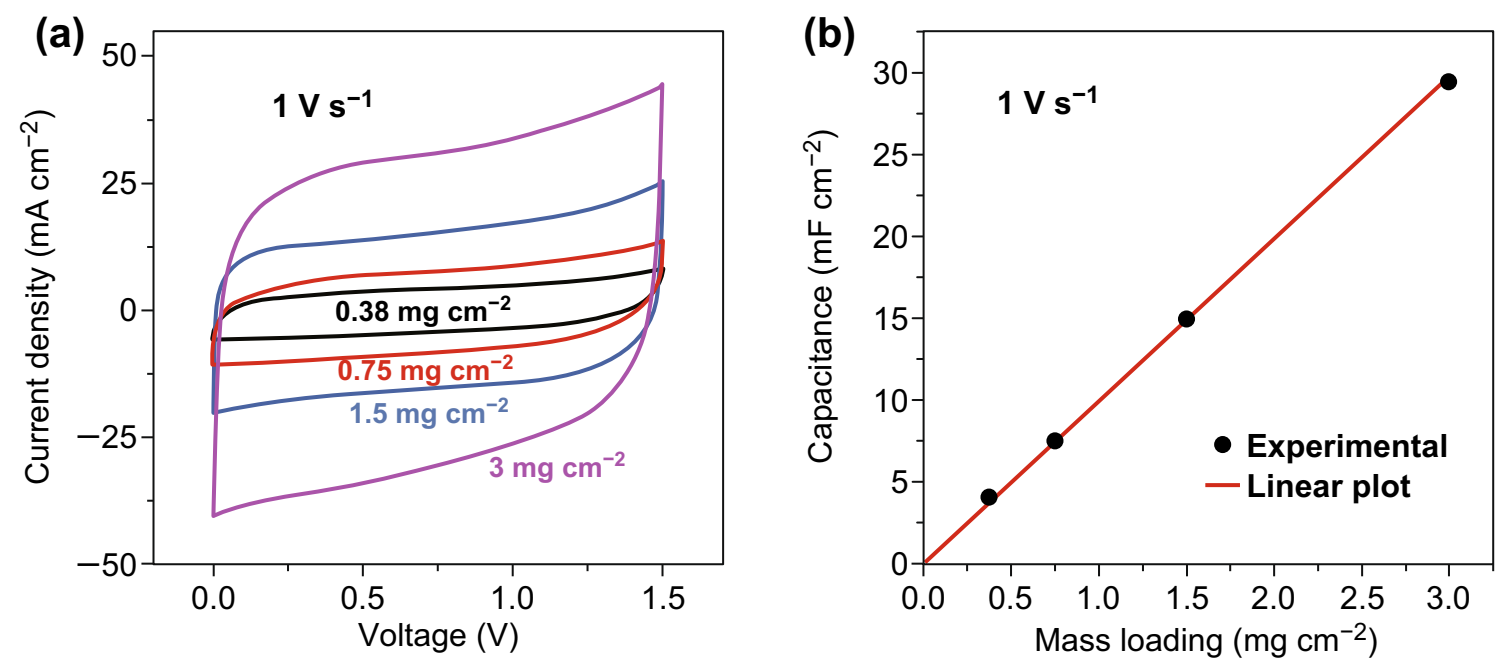

Fig. 7 a CV curves of TiN paper SSC with different mass loadings collected at a scan rate of $1 \mathrm{~V} \mathrm{~s}^{-1}$. b Areal capacitance of TiN paper SSC is plotted as a function of TiN mass loading. The linear solid line is a guide to the eye

$1 \mathrm{M} \mathrm{KOH}$ electrolyte, respectively (Fig. 6a-d). SEM images revealed that the porous structure of TiN nanobelts remained unchanged after cycling in $0.5 \mathrm{M} \mathrm{Na}_{2} \mathrm{SO}_{4}$ electrolyte. In contrast, the nanobelt morphology of TiN changed significantly after testing in $1 \mathrm{M} \mathrm{H}_{2} \mathrm{SO}_{4}$ and $1 \mathrm{M}$ $\mathrm{KOH}$ solutions (Fig. 6e-g). XPS spectra were collected to investigate the chemical nature of TiN electrode surface before and after cycling stability test. The $\mathrm{N} 1 \mathrm{~s}$ spectrum of TiN paper tested in $0.5 \mathrm{M} \mathrm{Na}_{2} \mathrm{SO}_{4}$ solution shows no obvious change, while the $\mathrm{N} 1 \mathrm{~s}$ peaks disappeared after cycling in $1 \mathrm{M} \mathrm{H}_{2} \mathrm{SO}_{4}$ or $1 \mathrm{M} \mathrm{KOH}$ electrolytes (Fig. S18). Ti 2p XPS spectra further showed that the signal of Ti-N and Ti-N-O decreased considerably after testing in $\mathrm{H}_{2} \mathrm{SO}_{4}$ or $\mathrm{KOH}$ solution, leaving only $\mathrm{Ti}-\mathrm{O}$ signals, while the Ti-N and Ti-N-O signals were not affected for TiN paper tested in $0.5 \mathrm{M} \mathrm{Na}_{2} \mathrm{SO}_{4}$. These results suggested that TiN papers were oxidized in both $\mathrm{H}_{2} \mathrm{SO}_{4}$ and $\mathrm{KOH}$ solutions, which are consistent with the previous reports $[25,63$, 64]. Titanium ions in TiN can be oxidized to soluble titanate ions $\left(\mathrm{HTiO}_{3}{ }^{-}\right)$and/or $\mathrm{TiO}_{2} \cdot \mathrm{H}_{2} \mathrm{O}$ in alkaline solution [63], while they can also be oxidized steadily to the trivalent state $\left(\mathrm{Ti}(\mathrm{OH})^{2+}\right)$ and tetravalent state $\left(\mathrm{Ti}(\mathrm{OH})_{2}^{2+}\right)$ at positive voltages in acidic solutions [64]. These results demonstrate the importance of selecting the sulfate-based neutral electrolyte for TiN electrodes and possibly other nitride electrodes. The outstanding cycling stability of TiN with zero decay in capacitance after 200,000 cycles in $0.5 \mathrm{M} \mathrm{Na}_{2} \mathrm{SO}_{4}$ is much better than the previous reports on metal nitride electrodes (Table $\mathrm{S} 1$ ), such as TiN nanowires on carbon cloth ( $82 \%$ after 15,000 cycles) [22], $\mathrm{Mo}_{2} \mathrm{~N}$ nanobelts/graphene (85.7\% after 4000 cycles) [65], $\mathrm{Nb}_{4} \mathrm{~N}_{5}$ nanobelts ( $80 \%$ after 1000 cycles) [51], VN/CNT composite ( $82 \%$ after 10,000 cycles) [61], and $\mathrm{Fe}_{2} \mathrm{~N} /$ graphene (92.9\% after 20,000 cycles) [21].

The filtration method offers not only an easy way to make paper-like electrode, but also the capability of controlling the electrode thickness and mass loading. We investigated the capacitive performance of TiN paper SSC with different mass loadings. As shown in Fig. 7a, TiN paper SSC retains a rectangular CV curves at a high scan rate of $1 \mathrm{~V} \mathrm{~s}^{-1}$ under different mass loadings. When the mass loading increased from 0.38 to $3 \mathrm{mg} \mathrm{cm}^{-2}$, the areal capacitance increases almost linearly, manifesting the gravimetric and volumetric capacitance are not significantly affected with the increased mass loading (Figs. 7b and S19). The thickness/mass loading-insensitive capacitive behavior makes the TiN paper electrode promising for practical energy storage devices.

\section{Conclusion}

In summary, we have fabricated a freestanding highly conductive and porous TiN paper electrode that can be operated at an ultrahigh scan rate of $100 \mathrm{~V} \mathrm{~s}^{-1}$ in a wide voltage window of $1.5 \mathrm{~V}$ in a $\mathrm{Na}_{2} \mathrm{SO}_{4}$ electrolyte and shows 
no capacitance decay in 200,000 charge/discharge cycles. Importantly, the TiN paper SSC exhibits an outstanding response time with a characteristic time constant of $4 \mathrm{~ms}$. This can be attributed to the high conductivity of TiN nanobelts and the efficient ion diffusion in the unique electrode architecture constructed with a network of mesoporous TiN nanobelts. We believe the paper-like electrode fabrication method can be applied to other metal nitride materials and provide an alternative way to make electrodes for ultrafastcharging supercapacitors.

Acknowledgements This work was supported by Merced nAnomaterials Center for Energy and Sensing (MACES), a NASA funded MIRO center, under award NNX15AQ01. We acknowledge Dr. Tom Yuzvinsky from UC Santa Cruz for assistance with SEM and the W. M. Keck Center for Nanoscale Optofluidics for use of the FEI Quanta 3D Dualbeam microscope. We also thank J. Hauser from the UC Santa Cruz for performing the XRD measurements (supported by the US NSF MRI grant, MRI-1126845).

Open Access This article is licensed under a Creative Commons Attribution 4.0 International License, which permits use, sharing, adaptation, distribution and reproduction in any medium or format, as long as you give appropriate credit to the original author(s) and the source, provide a link to the Creative Commons licence, and indicate if changes were made. The images or other third party material in this article are included in the article's Creative Commons licence, unless indicated otherwise in a credit line to the material. If material is not included in the article's Creative Commons licence and your intended use is not permitted by statutory regulation or exceeds the permitted use, you will need to obtain permission directly from the copyright holder. To view a copy of this licence, visit http://creativecommons.org/licenses/by/4.0/.

Electronic supplementary material The online version of this article (https://doi.org/10.1007/s40820-019-0340-7) contains supplementary material, which is available to authorized users.

\section{References}

1. H.S. Kim, J.B. Cook, H. Lin, J.S. Ko, S.H. Tolbert, V. Ozolins, B. Dunn, Oxygen vacancies enhance pseudocapacitive charge storage properties of $\mathrm{MoO}_{3-x}$. Nat. Mater. 16, 454-460 (2017). https://doi.org/10.1038/nmat4810

2. N.A. Kyeremateng, T. Brousse, D. Pech, Microsupercapacitors as miniaturized energy-storage components for on-chip electronics. Nat. Nanotechnol. 12, 7-15 (2017). https://doi. org/10.1038/nnano.2016.196

3. D. Feng, T. Lei, M. Lukatskaya, J. Park, Z. Huang et al., Robust and conductive two-dimensional metal-organic frameworks with exceptionally high volumetric and areal capacitance.
Nat. Energy 3, 30-36 (2018). https://doi.org/10.1038/s4156 0-017-0044-5

4. Y. Huang, Z. Tang, Z. Liu, J. Wei, H. Hu, C. Zhi, Toward enhancing wearability and fashion of wearable supercapacitor with modified polyurethane artificial leather electrolyte. Nano-Micro Lett. 10, 38 (2018). https://doi.org/10.1007/s4082 0-018-0191-7

5. H. Sun, L. Mei, J. Liang, Z. Zhao, C. Lee et al., Three-dimensional holey-graphene/niobia composite architectures for ultrahigh-rate energy storage. Science 356, 599-604 (2017). https://doi.org/10.1126/science.aam5852

6. V. Augustyn, J. Come, M. Lowe, J. Kim, P. Taberna et al., High-rate electrochemical energy storage through $\mathrm{Li}^{+}$intercalation pseudocapacitance. Nat. Mater. 12, 518-522 (2013). https://doi.org/10.1038/nmat3601

7. B. Yao, J. Zhang, T. Kou, Y. Song, T. Liu, Y. Li, Paper-based electrodes for flexible energy storage devices. Adv. Sci. 4, 1700107 (2017). https://doi.org/10.1002/advs.201700107

8. M.H. Yu, X.L. Feng, Thin-film electrode-based supercapacitors. Joule 3, 338-360 (2019). https://doi.org/10.1016/j.joule .2018 .12 .012

9. L. Yuan, B. Yao, B. Hu, K. Huo, W. Chen, J. Zhou, Polypyrrole-coated paper for flexible solid-state energy storage. Energy Environ. Sci. 6, 470-476 (2013). https://doi. org/10.1039/c2ee23977a

10. V. Augustyn, P. Simon, B. Dunn, Pseudocapacitive oxide materials for high-rate electrochemical energy storage. Energy Environ. Sci. 7, 1597-1614 (2014). https://doi.org/10.1039/ c3ee44164d

11. Y. Song, T. Liu, B. Yao, M. Li, T. Kou et al., Ostwald ripening improves rate capability of high mass loading manganese oxide for supercapacitors. ACS Energy Lett. 2, 1752-1759 (2017). https://doi.org/10.1021/acsenergylett.7000405

12. M. Zhang, Q. Zhou, J. Chen, X. Yu, L. Huang, Y. Li, C. Li, G. Shi, An ultrahigh-rate electrochemical capacitor based on solution-processed highly conductive PEDOT:PSS films for AC line-filtering. Energy Environ. Sci. 9, 2005-2010 (2016). https://doi.org/10.1039/C6EE00615A

13. N. Kurra, M.K. Hota, H.N. Alshareef, Conducting polymer micro-supercapacitors for flexible energy storage and Ac line-filtering. Nano Energy 13, 500-508 (2015). https://doi. org/10.1016/j.nanoen.2015.03.018

14. J.A. Lee, M.K. Shin, S.H. Kim, H.U. Cho, G.M. Spinks et al., Ultrafast charge and discharge biscrolled yarn supercapacitors for textiles and microdevices. Nat. Commun. 4, 1970 (2013). https://doi.org/10.1038/ncomms2970

15. D.T. Pham, T.H. Lee, D.H. Luong, F. Yao, A. Ghosh et al., Carbon nanotube-bridged graphene $3 \mathrm{~d}$ building blocks for ultrafast compact supercapacitors. ACS Nano 9, 2018-2027 (2015). https://doi.org/10.1021/nn507079x

16. G.H. Lee, J.W. Lee, J.I. Choi, S.J. Kim, Y.H. Kim, J.K. Kang, Ultrafast discharge/charge rate and robust cycle life for highperformance energy storage using ultrafine nanocrystals on the binder-free porous graphene foam. Adv. Funct. Mater. 26, 5139-5148 (2016). https://doi.org/10.1002/adfm.201601355 
17. Z.S. Wu, Z. Liu, K. Parvez, X. Feng, K. Mullen, Ultrathin printable graphene supercapacitors with AC line-filtering performance. Adv. Mater. 27, 3669-3675 (2015). https://doi. org/10.1002/adma.201501208

18. J. Lin, C. Zhang, Z. Yan, Y. Zhu, Z. Peng et al., 3-Dimensional graphene carbon nanotube carpet-based microsupercapacitors with high electrochemical performance. Nano Lett. 13, 72-78 (2013). https://doi.org/10.1021/nl3034976

19. R.H. Baughman, A.A. Zakhidov, W.A. de Heer, Carbon nanotubes-the route toward applications. Science 297, 787-792 (2002). https://doi.org/10.1126/science.1060928

20. Y. Zhu, S. Murali, M.D. Stoller, K.J. Ganesh, W. Cai et al., Carbon-based supercapacitors produced by activation of graphene. Science 332, 1537-1541 (2011). https://doi. org/10.1126/science. 1200770

21. C. Zhu, P. Yang, D. Chao, X. Wang, X. Zhang et al., All metal nitrides solid-state asymmetric supercapacitors. Adv. Mater. 27, 4566-4571 (2015). https://doi.org/10.1002/adma.20150 1838

22. X. Lu, G. Wang, T. Zhai, M. Yu, S. Xie et al., Stabilized TiN nanowire arrays for high-performance and flexible supercapacitors. Nano Lett. 12, 5376-5381 (2012). https://doi. org/10.1021/nl302761z

23. M.-S. Balogun, W. Qiu, W. Wang, P. Fang, X. Lu, Y. Tong, Recent advances in metal nitrides as high-performance electrode materials for energy storage devices. J. Mater. Chem. A 3, 1364-1387 (2015). https://doi.org/10.1039/C4TA05565A

24. X. Lu, T. Liu, T. Zhai, G. Wang, M. Yu et al., Improving the cycling stability of metal-nitride supercapacitor electrodes with a thin carbon shell. Adv. Energy Mater. 4, 1300994 (2014). https://doi.org/10.1002/aenm.201300994

25. G. Hasegawa, A. Kitada, S. Kawasaki, K. Kanamori, K. Nakanishi et al., Impact of electrolyte on pseudocapacitance and stability of porous titanium nitride (TiN) monolithic electrode. J. Electrochem. Soc. 162, A77-A85 (2015). https://doi. org/10.1149/2.0491501jes

26. B. Yao, L. Huang, J. Zhang, X. Gao, J. Wu et al., Flexible transparent molybdenum trioxide nanopaper for energy storage. Adv. Mater. 28, 6353-6358 (2016). https://doi. org/10.1002/adma.201600529

27. F. Zhang, T. Liu, M. Li, M. Yu, Y. Luo, Y. Tong, Y. Li, Multiscale pore network boosts capacitance of carbon electrodes for ultrafast charging. Nano Lett. 17, 3097-3104 (2017). https ://doi.org/10.1021/acs.nanolett.7b00533

28. M. Zukalova, J. Prochazka, Z. Bastl, J. Duchoslav, L. Rubacek, D. Havlicek, L. Kavan, Facile conversion of electrospun $\mathrm{TiO}_{2}$ into titanium nitride/oxynitride fibers. Chem. Mater. 22, 40454055 (2010). https://doi.org/10.1021/cm100877h

29. H.-C. Park, K.-H. Lee, Y.-W. Lee, S.-J. Kim, D.-M. Kim, M.-C. Kim, K.-W. Park, Mesoporous molybdenum nitride nanobelts as an anode with improved electrochemical properties in lithium ion batteries. J. Power Sources 269, 534-541 (2014). https://doi.org/10.1016/j.jpowsour.2014.07.009

30. L. Yan, G. Chen, S. Tan, M. Zhou, G. Zou et al., Titanium oxynitride nanoparticles anchored on carbon nanotubes as energy storage materials. ACS Appl. Mater. Interfaces 7, 24212-24217 (2015). https://doi.org/10.1021/acsami.5b07630

31. Z. Zhang, J.B.M. Goodall, D.J. Morgan, S. Brown, R.J.H. Clark et al., Photocatalytic activities of N-doped nano-titanias and titanium nitride. J. Eur. Ceram. Soc. 29, 2343-2353 (2009). https://doi.org/10.1016/0010-938X(93)90165-D

32. M. Harb, P. Sautet, P. Raybaud, Origin of the enhanced visible-light absorption in n-doped bulk anatase $\mathrm{TiO}_{2}$ from firstprinciples calculations. J. Phys. Chem. C 115, 19394-19404 (2011). https://doi.org/10.1021/jp204059q

33. P. Yang, D. Chao, C. Zhu, X. Xia, Y. Zhang et al., Ultrafastcharging supercapacitors based on corn-like titanium nitride nanostructures. Adv. Sci. 3, 1500299 (2016). https://doi. org/10.1002/advs.201500299

34. S. Oktay, Z. Kahraman, M. Urgen, K. Kazmanli, XPS investigations of tribolayers formed on $\mathrm{TiN}$ and $(\mathrm{Ti}, \mathrm{Re}) \mathrm{N}$ coatings. Appl. Surf. Sci. 328, 255-261 (2015). https://doi. org/10.1016/j.apsusc.2014.12.023

35. R. Chandrasekaran, Y. Soneda, J. Yamashita, M. Kodama, H. Hatori, Preparation and electrochemical performance of activated carbon thin films with polyethylene oxide-salt addition for electrochemical capacitor applications. J. Solid State Electrochem. 12, 1349-1355 (2008). https://doi.org/10.1007/ s10008-008-0559-6

36. Y. Xu, Z. Lin, X. Zhong, X. Huang, N.O. Weiss, Y. Huang, X. Duan, Holey graphene frameworks for highly efficient capacitive energy storage. Nat. Commun. 5, 4554 (2014). https://doi. org/10.1038/ncomms5554

37. L. Huang, B. Yao, J. Sun, X. Gao, J. Wu et al., Highly conductive and flexible molybdenum oxide nanopaper for high volumetric supercapacitor electrode. J. Mater. Chem. A 5, 2897-2903 (2017). https://doi.org/10.1039/C6TA10433A

38. K.S. Kim, Y. Zhao, H. Jang, S.Y. Lee et al., Large-scale pattern growth of graphene films for stretchable transparent electrodes. Nature 457, 706-710 (2009). https://doi.org/10.1038/ nature 07719

39. U.N. Maiti, J. Lim, K.E. Lee, W.J. Lee, S.O. Kim, Threedimensional shape engineered, interfacial gelation of reduced graphene oxide for high rate, large capacity supercapacitors. Adv. Mater. 26, 615-619 (2014). https://doi.org/10.1002/ adma.201303503

40. L. Hu, J.W. Choi, Y. Yang, S. Jeong, F. La Mantia, L.F. Cui, Y. Cui, Highly conductive paper for energy-storage devices. Proc. Natl. Acad. Sci. USA 106, 21490-21494 (2009). https ://doi.org/10.1073/pnas.0908858106

41. L. Yuan, X. Xiao, T. Ding, J. Zhong, X. Zhang, Y. Shen et al., Paper-based supercapacitors for self-powered nanosystems. Angew. Chem. Int. Ed. 51, 4934-4938 (2012). https://doi. org/10.1002/anie.201109142

42. Z. Li, G. Ma, R. Ge, F. Qin, X. Dong et al., Free-standing conducting polymer films for high-performance energy devices. Angew. Chem. Int. Ed. 55, 979-982 (2016). https:// doi.org/10.1002/anie.201509033

43. T. Zheng, M.H. Tahmasebi, B. Li, Y. Li, S. Ran et al., Sputtered titanium nitride films on titanium foam substrates as 
electrodes for high-power electrochemical capacitors. ChemElectroChem 5, 2199-2207 (2018). https://doi.org/10.1002/ celc. 201800467

44. F. Grote, H. Zhao, Y. Lei, Self-supported carbon coated TiN nanotube arrays: innovative carbon coating leads to an improved cycling ability for supercapacitor applications. J. Mater. Chem. A 3, 3465-3470 (2015). https://doi.org/10.1039/ C4TA05905K

45. P. Sun, R. Lin, Z. Wang, M. Qiu, Z. Chai et al., Rational design of carbon shell endows TiN@C nanotube based fiber supercapacitors with significantly enhanced mechanical stability and electrochemical performance. Nano Energy 31, 432-440 (2017). https://doi.org/10.1016/j.nanoen.2016.11.052

46. E. Kao, C. Yang, R. Warren, A. Kozinda, L. Lin, ALD titanium nitride on vertically aligned carbon nanotube forests for electrochemical supercapacitors. Sens. Actuators A-Phys. 240, 160-166 (2016). https://doi.org/10.1016/j.sna.2016.01.044

47. L. Demarconnay, E. Raymundo-Piñero, F. Béguin, A symmetric carbon/carbon supercapacitor operating at $1.6 \mathrm{~V}$ by using a neutral aqueous solution. Electrochem. Commun. 12, 12751278 (2010). https://doi.org/10.1016/j.elecom.2010.06.036

48. Q. Gao, L. Demarconnay, E. Raymundo-Pinero, F. Beguin, Exploring the large voltage range of carbon/carbon supercapacitors in aqueous lithium sulfate electrolyte. Energy Environ. Sci. 5, 9611-9617 (2012). https://doi.org/10.1039/c2ee2 $2284 \mathrm{a}$

49. T. Lin, I. Chen, F. Liu, C. Yang, H. Bi, F. Xu, F. Huang, Nitrogen-doped mesoporous carbon of extraordinary capacitance for electrochemical energy storage. Science 350, 1508-1513 (2015). https://doi.org/10.1126/science.aab3798

50. K. Fic, G. Lota, M. Meller, E. Frackowiak, Novel insight into neutral medium as electrolyte for high-voltage supercapacitors. Energy Environ. Sci. 5, 5842-5850 (2012). https://doi. org/10.1039/c1ee02262h

51. H. Cui, G. Zhu, X. Liu, F. Liu, Y. Xie et al., Niobium nitride $\mathrm{Nb}_{4} \mathrm{~N}_{5}$ as a new high-performance electrode material for supercapacitors. Adv. Sci. 2, 1500126 (2015). https://doi. org/10.1002/advs.201500126

52. B. Gao, X. Xiao, J. Su, X. Zhang, X. Peng, J. Fu, P.K. Chu, Synthesis of mesoporous niobium nitride nanobelt arrays and their capacitive properties. Appl. Surf. Sci. 383, 57-63 (2016). https://doi.org/10.1016/j.apsusc.2016.04.173

53. X. Lu, M. Yu, T. Zhai, G. Wang, S. Xie et al., High energy density asymmetric quasi-solid-state supercapacitor based on porous vanadium nitride nanowire anode. Nano Lett. 13, 2628-2633 (2013). https://doi.org/10.1021/nl400760a

54. Y. Liu, R. Xiao, Y. Qiu, Y. Fang, P. Zhang, Flexible advanced asymmetric supercapacitors based on titanium nitride-based nanowire electrodes. Electrochim. Acta 213, 393-399 (2016). https://doi.org/10.1016/j.electacta.2016.06.166
55. P. Xu, T. Gu, Z. Cao, B. Wei, J. Yu et al., Carbon nanotube fiber based stretchable wire-shaped supercapacitors. Adv. Energy Mater. 4, 1300759-1300764 (2014). https://doi. org/10.1002/aenm.201300759

56. W. Bao, A.K. Mondal, J. Xu, C. Wang, D. Su, G. Wang, 3D hybrid-porous carbon derived from carbonization of metal organic frameworks for high performance supercapacitors. J. Power Sources 325, 286-291 (2016). https://doi.org/10.1016/j. jpowsour.2016.06.037

57. V. Presser, L. Zhang, J.J. Niu, J. McDonough, C. Perez, H. Fong, Y. Gogotsi, Flexible nano-felts of carbide-derived carbon with ultra-high power handling capability. Adv. Energy Mater. 1, 423-430 (2011). https://doi.org/10.1002/aenm.20110 0047

58. M. El-Kady, V. Strong, S. Dubin, R. Kaner, Laser scribing of high-performance and flexible graphene-based electrochemical capacitors. Science 335, 1326-1330 (2012). https://doi. org/10.1126/science. 1216744

59. D. Pech, M. Brunet, H. Durou, P. Huang, V. Mochalin, Y. Gogotsi, P.L. Taberna, P. Simon, Ultrahigh-power micrometresized supercapacitors based on onion-like carbon. Nat. Nanotechnol. 5, 651-654 (2010). https://doi.org/10.1038/nnano .2010 .162

60. T. Liu, Z. Zhou, Y. Guo, D. Guo, G. Liu, Block copolymer derived uniform mesopores enable ultrafast electron and ion transport at high mass loadings. Nat. Commun. 10, 675 (2019). https://doi.org/10.1038/s41467-019-08644-w

61. X. Xiao, X. Peng, H. Jin, T. Li, C. Zhang et al., Freestanding mesoporous VN/CNT hybrid electrodes for flexible all-solidstate supercapacitors. Adv. Mater. 25, 5091-5097 (2013). https ://doi.org/10.1002/adma.201301465

62. Y. Xie, Y. Wang, H. Du, Electrochemical capacitance performance of titanium nitride nanoarray. Mater. Sci. Eng. B 178, 1443-1451 (2013). https://doi.org/10.1016/j. mseb.2013.09.005

63. C.F. Windisch, J.W. Virden, S.H. Elder, J. Liu, M.H. Engelhard, Electrochemistry of TiN in $6 \mathrm{M} \mathrm{KOH}$ Solution. J. Electrochem. Soc. 145, 1211-1218 (1998). https://doi. org/10.1149/1.1838441

64. S.D. Chyou, H.C. Shih, T.T. Chen, On the corrosion characterization of titanium nitride in sulfuric acid solution. Corros. Sci. 35, 337-347 (1993). https://doi.org/10.1016/0010938X(93)90165-D

65. G.Q. Ma, Z. Wang, B. Gao, T.P. Ding, Q.Z. Zhong et al., Multilayered paper-like electrodes composed of alternating stacked mesoporous $\mathrm{Mo}_{2} \mathrm{~N}$ nanobelts and reduced graphene oxide for flexible all-solid-state supercapacitors. J. Mater. Chem. A 3, 14617-14624 (2015). https://doi.org/10.1039/c5ta02851e 